\title{
Prenatal Opioid Exposure and ADHD Childhood Symptoms: A Meta-Analysis
}

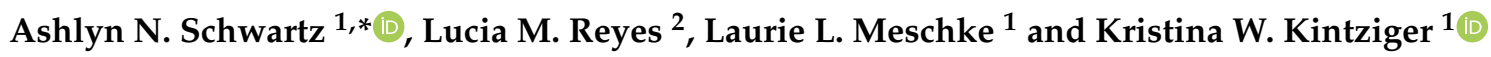 \\ 1 The Department of Public Health, The University of Tennessee, Knoxville, TN 37996, USA; \\ llmeschke@utk.edu (L.L.M.); kkintzig@utk.edu (K.W.K.) \\ 2 The Department of Child and Family Studies, The University of Tennessee, Knoxville, TN 37996, USA; \\ luciagmiranda@gmail.com \\ * Correspondence: aschwa13@vols.utk.edu; Tel.: +1-678-447-3930
}

Citation: Schwartz, A.N.; Reyes,

L.M.; Meschke, L.L.; Kintziger, K.W. Prenatal Opioid Exposure and ADHD Childhood Symptoms: A Meta-Analysis. Children 2021, 8, 106. https://doi.org/10.3390/ children8020106

Academic Editor: Bruce Dick Received: 22 December 2020 Accepted: 31 January 2021 Published: 4 February 2021

Publisher's Note: MDPI stays neutral with regard to jurisdictional claims in published maps and institutional affiliations.

Copyright: (c) 2021 by the authors. Licensee MDPI, Basel, Switzerland. This article is an open access article distributed under the terms and conditions of the Creative Commons Attribution (CC BY) license (https:// creativecommons.org/licenses/by/ $4.0 /)$.

\begin{abstract}
To systematically investigate the association between prenatal opioid exposure (POE) and attention-deficit hyperactivity disorder (ADHD) symptoms in children 2-18 years old, studies were searched using PubMed, CINAHL, PsycINFO, and Web of Science from January of 1950 to October of 2019. Inclusion criteria were observational studies reporting ADHD symptoms of children with POE compared with non-exposed children or normative data. The study protocol was registered with PROSPERO: CRD42018115967. Two independent reviewers extracted data on hyperactivity/impulsivity, inattention symptoms, ADHD combined subscale symptoms, and sample characteristics. Of 223 articles screened, seven met the inclusion criteria. Data represent 319 children with POE and 1308 non-exposed children from 4.3 to 11.2 mean years from five countries. POE was positively associated with childhood hyperactivity/impulsivity ( $d=1.40 ; 95 \% \mathrm{CI}, 0.49-2.31$; $p=0.003)$, inattention $(\mathrm{d}=1.35 ; 95 \% \mathrm{CI}, 0.69-2.01 ; p<0.0001)$, and combined ADHD symptoms scores ( $d=1.27 ; 95 \% \mathrm{CI}=0.79-1.75 ; p<0.0001)$. POE was positively associated with ADHD combined symptom scores at preschool $(\mathrm{d}=0.83,95 \% \mathrm{CI}, 0.57,1.09 ; p<0.0001)$ and school age $(\mathrm{d}=1.45,95 \% \mathrm{CI}$, 0.85 to 2.04; $p<0.0001$ ). Results suggest increased risk of ADHD symptoms during school age. Future research is needed to clarify the relationship between biological, social, and environmental risk and ADHD symptoms for children who experienced POE.
\end{abstract}

Keywords: prenatal opioid exposure; attention deficit hyperactivity disorder; neonatal abstinence syndrome; neonatal opioid withdrawal syndrome

\section{Introduction}

Opioid use amongst reproductive-age women is increasing globally [1,2] and has been associated with infant risk, including neonatal abstinence syndrome (NAS) [3-6], low birth weight [7], preterm birth [8], and altered neonatal brain development [9]. Consequently, there has been a five-fold increase in NAS within the last two decades [3]. Infants with NAS burden the healthcare system, with longer length of hospital stay and increased costs [10]. Pregnant women with opioid dependency represent a vulnerable group of women who have a history of family socioeconomic adversity, child custody loss, and many psychiatric, social, and obstetric needs [11,12]. Thus, children with prenatal opioid exposure (POE) face disproportionate risks. In addition to the neonatal concerns related to POE, some evidence indicates that POE may confer neurodevelopmental risk that endures beyond infancy. A meta-analysis of five studies of POE children's neurobehavior by Baldacchino and colleagues found significant impairments for cognitive, psychomotor, and behavioral outcomes compared to non-exposed controls in infant and preschool children [13]. Furthermore, in a meta-analysis by Monnelly and colleagues, six of seven studies found that children with POE had increased behavioral problems compared to non-exposed controls of children less than 2 years [14]. 
Most consistently in the literature, POE has been associated with behavioral issues in early childhood, primarily with symptoms of attention-deficit hyperactivity disorder (ADHD) [15-23]. For instance, in a cohort of children born in Norway, POE predicted elevated levels of inattention and impulsivity at both 2 and 4.5 years compared to nonexposed peers [16]. Similarly, a longitudinal study in New Zealand revealed that children with POE had increased inattention and hyperactivity during preschool years compared to non-exposed controls [15]. However, confounders associated with increased risk for ADHD (e.g., care disruption and prenatal polydrug exposure) were evident in both studies [15,16], thus limiting conclusions about the unique role of POE on ADHD symptoms. Less is known about whether ADHD symptoms persist into the school-age period. In small cross-sectional studies, POE has been linked with elevated hyperactivity at 8.5 years [22], poorer sustained attention in boys 7-12 years [24], and higher inattention and impulsivity at 8.5 years [21]. Behavioral ranking scores of Swedish children with POE have corresponded to normative ADHD scores, including symptoms of activity, inattention, and combined subscale scores of children, although the sample size was limited to 16 children [17]. Utilizing cutoff scores for ADHD in behavioral scales, a longitudinal study in Israel reported that over half of the children with POE raised in the biological mother's home had ADHD and a quarter of adopted children with POE had ADHD [20]. These findings also appear to indicate a pathway between POE and ADHD symptoms, where adoptive homes may mitigate but not eliminate long-term risk. In sum, even though the cognitive and motor outcomes of older children with POE have been assessed [25], no studies have reviewed POE and ADHD associations in older children.

To fill these gaps in the literature, this meta-analysis aimed to systematically investigate the strength of the association between POE and ADHD symptoms in children, ages 2-18 years. We investigated symptoms of hyperactivity/impulsivity and inattention separately as well as combined and performed subgroup analyses of preschool (4.3-4.5 mean years) and school-age children (5.4-11.2 mean years). We hypothesized that children 2-18 years with POE will have greater mean scores of inattentive, hyperactive/impulsive, and combined symptoms compared to their non-exposed peers.

\section{Materials and Methods}

This meta-analysis was registered with the International Prospective Register of Systematic Reviews (PROSPERO), registration number CRD42018115967 and was conducted using an a priori protocol following the Preferred Reporting Items for Systematic Reviews and Meta-Analyses (PRISMA) guidelines (Appendix A) [26].

To be included in this meta-analysis, studies had to meet the following inclusion criteria: (1) observational study design; (2) all participants with POE (or polydrug exposure including opioids) with a mean age of 2-18 years at follow-up; (3) inclusion of a control or comparison group or use of an established instrument with normative data available; (4) hyperactive/impulsive and/or inattentive behaviors reported via caregiver or teacher ratings, and (5) sufficient statistical information reported or provided upon contacting lead authors. There were no geographical restrictions, and language was restricted to English.

A literature search was conducted independently by the first and second authors for observational studies of ADHD symptoms in children aged 2-18 years with POE, published between January 1950 and October 2019 by searching the following electronic databases: CINAHL, PubMed, PsycINFO, and Web of Science. Filters for the search included limiting articles to human studies and the English language. The finalized search was run on October 20, 2019 and an additional search was re-run before the final analyses to look for additional studies for inclusion. The following keywords were used: ((opioid OR opiate OR heroin OR methadone OR buprenorphine OR NAS OR MAT OR suboxone OR infant withdrawal syndrome OR Subutex OR OMT OR fentanyl OR morphine OR tramadol OR codeine OR hydrocodone OR oxycodone) AND (in utero OR prenatal) AND (infan* OR toddler OR child* OR adolescen*) AND (ADHD OR hyperactivity OR impulsivity OR attention OR ADD OR neurobehavior OR executive function)). 
A standardized data extraction tool provided the following information when available: child impulsivity/hyperactivity, inattention, and combined ADHD subscale scores, outcome assessment mean age, number of participants, type of opioid exposure, polydrug exposure, method to identify opioid exposure, NAS diagnosis, care disruption, socioeconomic status, measurement instrument used, respondent of instrument, and country of study. Means and standard deviations were extracted on primary outcome measures.

A quantitative synthesis for eligible studies is included using aggregate data with the Comprehensive Meta-Analysis (CMA) Software (V.3). Means and standard deviations were transformed to standardized scores and mean effect sizes (SMD). An effect size was considered small (0.3-0.4), moderate (0.5-0.7), or large ( $>0.8)$ according to Cohen's $\mathrm{d}$ [27]. Assessments for heterogeneity between studies in effect measures were conducted using Higgins $\mathrm{I}^{2}$, where $\mathrm{I}^{2}$ values greater than $50 \%$ were considered significant heterogeneity [27]. Publication bias was visually assessed by reviewing funnel plots for asymmetry and with the Egger test. As eligible studies had large heterogeneity, a random-effect meta-analysis with standardized mean differences were calculated for reported hyperactivity/impulsivity, inattention, and combined ADHD subscale symptoms. Subgroup analyses for age (preschool, 4.3-4.5 mean years; school age, 5.4-11.2 mean years) and control/comparison group (healthy control/comparison, healthy children from mothers without opioid use disorder (OUD); environmental control/comparison, children raised by a father with OUD or children raised in a low socioeconomic status with environmental deprivation or neglect) were conducted for reported combined ADHD subscale symptoms using a random-effect meta-analysis.

The Newcastle-Ottawa Scale for case control and cohort studies was used to assess the risk of bias in included studies and was rated by two independent reviewers [28]. Disagreements were discussed between the first and second author. The Newcastle-Ottawa Scale has a range of possible scores from 0 to 9 , with higher scores indicating a better study quality. A score of 3 or less for a study was considered low quality. A sensitivity analysis was conducted using only studies above this threshold to assess if low quality studies influenced the effect size.

\section{Results}

\subsection{Study Selection}

Overall, 264 articles were included in the literature search. After removal of $108 \mathrm{du}-$ plicates, 156 articles were available for title, abstract, and full-text screening. Reasons for article exclusion at each stage are shown in Figure 1. Title and abstract review removed 119 articles and after full-text review, 30 articles were further removed. In total, seven studies were left for inclusion in the meta-analysis. The overall agreement after title and abstract selection was $94 \%$ and Cohen's $\mathrm{k}(0.85)$ and $93 \%(0.81)$ at the full text retrieval stage.

Due to the nature of longitudinal follow-up studies, there were five instances where multiple reports were published on different time points of the same cohort $[16,18,19,29,30]$. In these cases, only one study per primary outcome was included per cohort of children to avoid bias by counting the same participants twice. In such instances, publications with the largest sample sizes, including the highest $n$ for primary outcomes, were included in this meta-analysis. When one study published on multiple measures for the same outcome variable, the measure with the largest sample size and with the highest validity and reliability was chosen $(n=1)$ [31]. If a study had more than one control group, the control group whose characteristics most closely represented the non-exposed normative population was chosen $(n=1)$ [20]. Overall, this resulted in excluding five studies in the full-text review stage that met one or more of these exclusion criteria $[16,18,19,29,30]$. 


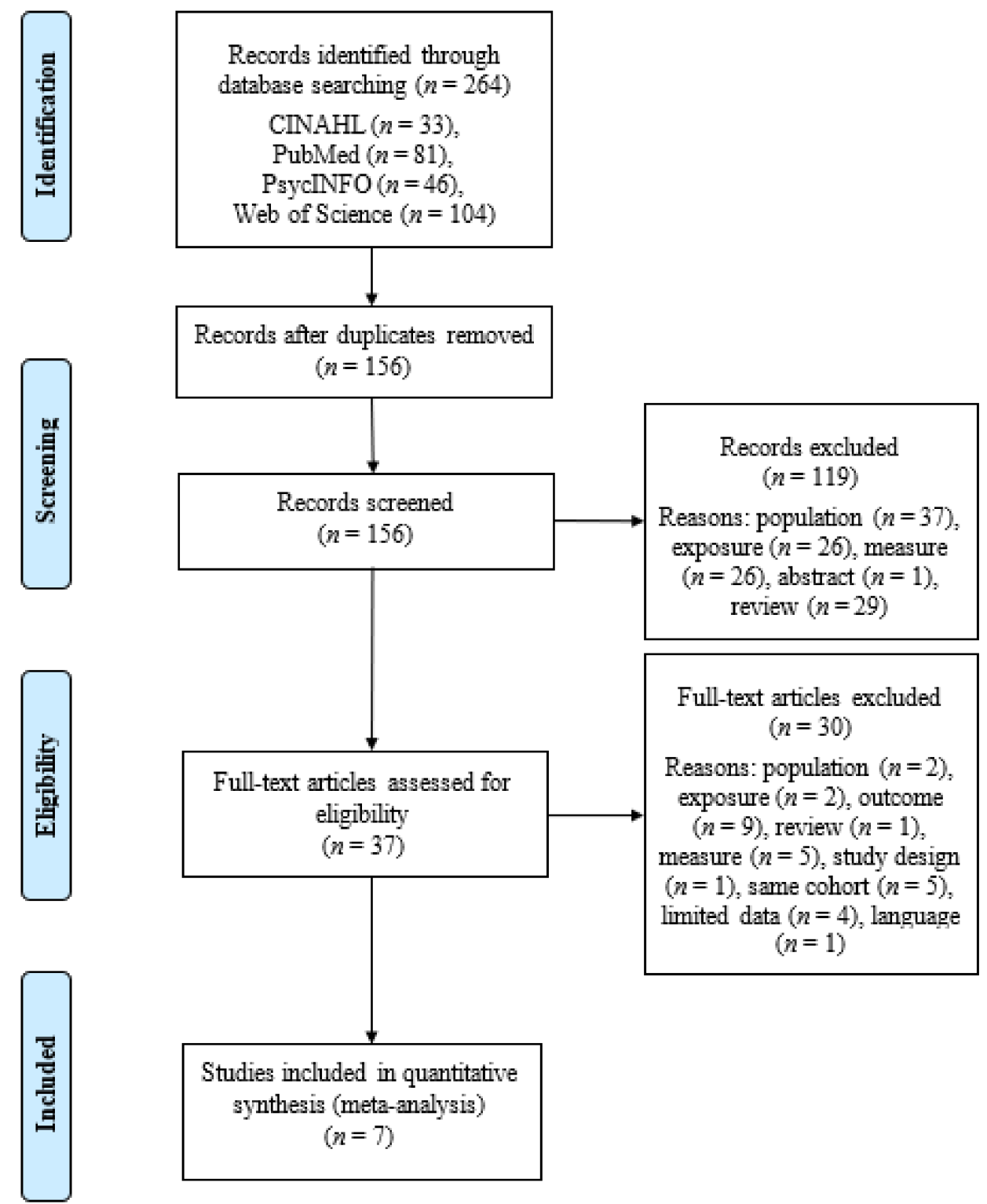

Figure 1. PRISMA flow diagram of literature search.

\subsection{Study Characteristics}

Details regarding characteristics of eligible studies are summarized in Tables 1 and 2. The seven studies included 319 children with POE and 1308 non-exposed children ranging from 4.2 to 14.2 years of age from Norway [23,31,32], Sweden [17], New Zealand [15], United States [21], and Israel [20]. Studies were published from 1988 to 2018. Polydrug exposure was present in all studies and included the following: tobacco/nicotine, alcohol, marijuana, stimulants, amphetamines, antipsychotic medications, antidepressants, benzodiazepines, opioids/narcotics, and illicit drugs. The main opioid exposure being heroin in one study [23], methadone and/or buprenorphine in four studies [15,17,31,32], and a combination of heroin and methadone in two studies $[20,21]$. The method to identify opioid exposure included self-report [15,20,23,32], methadone and hospital records [21,23,31], social records [23], and maternal toxicology and infant meconium [15,17]. A change in the primary caregiver, defined as care disruption, was present in all but one study [21], ranging from $14.3 \%$ to $98 \%$. In three studies $[15,23,32]$, SES significantly differed between the exposed and non-exposed children. NAS was reported in five of the seven studies $[15,17,23,31,32]$, with reported rates ranging from $17.8 \%$ to $88.2 \%$. 
Table 1. Descriptive characteristics of (a) hyperactivity/impulsivity and (b) inattention outcomes.

\begin{tabular}{|c|c|c|c|c|c|c|c|c|c|c|c|c|}
\hline \multicolumn{13}{|c|}{ (a) Hyperactivity/Impulsivity } \\
\hline & & & \multicolumn{7}{|c|}{ POE Children } & \multicolumn{3}{|c|}{ Non-Exposed Children } \\
\hline $\begin{array}{c}\text { Study, } \\
\text { Place of Birth }\end{array}$ & $\begin{array}{l}\text { Measure } \\
\text { * Subscale } \\
\text { (Reporter) }\end{array}$ & $\begin{array}{c}\text { NAS } \\
(\%)\end{array}$ & $\begin{array}{l}\text { Polydrug } \\
\text { Exposure }\end{array}$ & SES & $\begin{array}{l}\text { Out Home } \\
(\%)\end{array}$ & POE & $\begin{array}{l}\text { Age at Test } \\
\text { M (SD) }\end{array}$ & $\begin{array}{l}\text { Outcome } \\
\text { M (SD) }\end{array}$ & $n$ & $\begin{array}{l}\text { Age at Test } \\
\text { M (SD) }\end{array}$ & $\begin{array}{l}\text { Outcome } \\
\text { M (SD) }\end{array}$ & $n$ \\
\hline $\begin{array}{c}\text { Konijnenberg, } 2015 \text { [32] } \\
\text { Norway }\end{array}$ & $\begin{array}{l}\text { BRIEF-P * } \\
\text { Inhibit } \\
\text { (CG) }\end{array}$ & 50 & $\begin{array}{c}\text { Tobacco, alcohol, } \\
\text { marijuana, } \\
\text { amphetamine, } \\
\text { benzodiazepine, } \\
\text { opioids, MTD, BUP }\end{array}$ & Yes & 14.3 & MTD/BUP & $4.4(0.1)$ & $55.97(11.1)$ & 35 & $4.3(0.1)$ & $47.42(7.1)$ & 31 \\
\hline $\begin{array}{l}\text { Davis, } 1988[21] \\
\text { United States }\end{array}$ & $\begin{array}{c}\text { Burk's } \\
\text { * Impulse Control } \\
\text { (CG) }\end{array}$ & N/A & $\begin{array}{l}\text { Combination of } \\
\text { narcotics }\end{array}$ & No & $\mathrm{N} / \mathrm{A}$ & Heroin/MTD & $8.5(2.5)$ & $14.71(7.9)$ & 28 & $11.2(3.0)$ & $8.43(3.7)$ & 28 \\
\hline $\begin{array}{l}\text { Sandtorv, } 2018 \text { [31] } \\
\text { Norway }\end{array}$ & $\begin{array}{c}\text { SNAP- IV } \\
{ }^{*} \text { Hyper/ impulsivity } \\
\text { (CG) }\end{array}$ & 44 & Number of illicit drugs & NR & NR & OMT & $10.4(2.2)$ & $8.86(5.3)$ & 57 & $10.3(2.0)$ & $1.42(2.4)$ & 171 \\
\hline \multicolumn{13}{|c|}{ (b) Inattention } \\
\hline $\begin{array}{l}\text { Konijnenberg, } 2015 \text { [32] } \\
\text { Norway }\end{array}$ & $\begin{array}{c}\text { BRIEF-P } \\
* \text { Working Memory } \\
\text { (CG) }\end{array}$ & 50 & $\begin{array}{c}\text { Tobacco, alcohol, } \\
\text { marijuana, } \\
\text { amphetamine, } \\
\text { benzodiazepine, } \\
\text { opioids, MTD, BUP }\end{array}$ & Yes & 14.3 & MTD/BUP & $4.4(0.1)$ & $57.46(11.4)$ & 35 & $4.3(0.1)$ & $49.13(9.9)$ & 31 \\
\hline $\begin{array}{l}\text { Davis, } 1988 \text { [21] } \\
\text { United States }\end{array}$ & $\begin{array}{l}\text { Burk's } \\
\text { * Attention } \\
\text { (CG) }\end{array}$ & N/A & $\begin{array}{l}\text { Combination of } \\
\text { narcotics }\end{array}$ & No & $\mathrm{N} / \mathrm{A}$ & Heroin/MTD & $8.5(2.5)$ & $15.18(6.3)$ & 28 & $11.2(3.0)$ & $8.0(2.5)$ & 28 \\
\hline $\begin{array}{l}\text { Sandtorv, } 2018 \text { [31] } \\
\text { Norway }\end{array}$ & $\begin{array}{l}\text { SNAP- IV } \\
\text { * Inattention } \\
\text { (CG) }\end{array}$ & 44 & Number of illicit drugs & NR & NR & OMT & $10.4(2.2)$ & $10.79(4.6)$ & 57 & $10.3(2.0)$ & $2.72(3.5)$ & 171 \\
\hline $\begin{array}{l}\text { Nygaard, } 2016 \text { [23] } \\
\text { Norway }\end{array}$ & $\begin{array}{c}\text { CBCL } \\
\text { * Attention } \\
\text { (CG) }\end{array}$ & 79 & $\begin{array}{c}\text { Tobacco, opiates, } \\
\text { cannabis, } \\
\text { amphetamines, } \\
\text { benzodiazepines, } \\
\text { alcohol, antipsychotic }\end{array}$ & Yes & 72 & Heroin & $8.6(0.6)$ & $5.1(4.2)$ & 57 & $8.7(0.5)$ & $1.7(4.9)$ & 47 \\
\hline
\end{tabular}

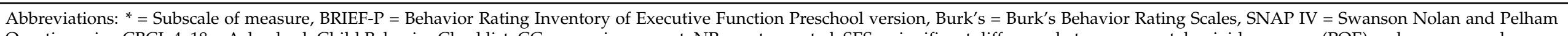

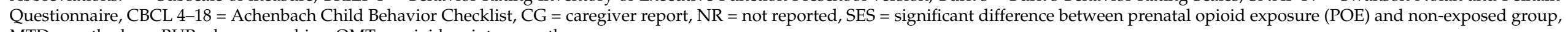

$\mathrm{MTD}=$ methadone, $\mathrm{BUP}=$ buprenorphine, $\mathrm{OMT}=$ opioid maintenance therapy. 
Table 2. Descriptive characteristics of combined attention-deficit hyperactivity disorder (ADHD) subscale outcomes.

\begin{tabular}{|c|c|c|c|c|c|c|c|c|c|c|c|c|}
\hline \multirow[b]{2}{*}{ Study, Place of Birth } & \multirow[b]{2}{*}{$\begin{array}{l}\text { Measure } \\
\text { (Reporter) }\end{array}$} & \multirow[b]{2}{*}{$\begin{array}{l}\text { NAS } \\
(\%)\end{array}$} & \multirow[b]{2}{*}{$\begin{array}{l}\text { Polydrug } \\
\text { Exposure }\end{array}$} & \multicolumn{6}{|c|}{ POE Children } & \multicolumn{3}{|c|}{ Non-Exposed Children } \\
\hline & & & & SES & $\begin{array}{l}\text { Out Home } \\
(\%)\end{array}$ & POE & $\begin{array}{l}\text { Age at Test } \\
\text { M (SD) }\end{array}$ & $\begin{array}{l}\text { Outcome M } \\
\text { (SD) }\end{array}$ & $n$ & $\begin{array}{l}\text { Age at Test } \\
\text { M (SD) }\end{array}$ & $\begin{array}{l}\text { Outcome M } \\
\text { (SD) }\end{array}$ & $n$ \\
\hline $\begin{array}{c}\text { Konijnenberg, } 2015 \text { [32] } \\
\text { Norway }\end{array}$ & $\begin{array}{l}\text { BRIEF-P } \\
\text { (CG) }\end{array}$ & 50 & $\begin{array}{c}\text { Tobacco, alcohol, } \\
\text { marijuana, } \\
\text { amphetamine, } \\
\text { benzodiazepine, } \\
\text { opioids, MTD, BUP }\end{array}$ & Yes & 14.3 & MTD/ BUP & $4.4(0.1)$ & $113.43(22.4)$ & 35 & $4.3(0.1)$ & $96.55(17.1)$ & 31 \\
\hline $\begin{array}{l}\text { Davis, } 1988[21] \\
\text { United States }\end{array}$ & $\begin{array}{l}\text { Burk's } \\
\text { (CG) }\end{array}$ & NR & $\begin{array}{l}\text { Combination of } \\
\text { narcotics }\end{array}$ & No & $\mathrm{N} / \mathrm{A}$ & Heroin/MTD & $8.5(2.5)$ & $29.9(13.5)$ & 28 & $11.2(3.0)$ & $16.43(6.2)$ & 28 \\
\hline $\begin{array}{c}\text { Sandtorv, } 2018 \text { [31] } \\
\text { Norway }\end{array}$ & $\begin{array}{l}\text { SNAP- IV } \\
\text { (CG) }\end{array}$ & 44 & Number of illicit drugs & NR & NR & OMT & $10.4(2.2)$ & $19.65(9.1)$ & 57 & $10.3(2.0)$ & $4.13(5.4)$ & 171 \\
\hline $\begin{array}{l}\text { Nygaard, } 2016 \text { [23] } \\
\text { Norway }\end{array}$ & $\begin{array}{l}\text { ADHD Rating Scale } \\
\text { (CG) }\end{array}$ & 79 & $\begin{array}{c}\text { Tobacco, opiates, } \\
\text { cannabis, } \\
\text { amphetamines, } \\
\text { benzodiazepines, } \\
\text { alcohol, antipsychotic }\end{array}$ & Yes & 72 & Heroin & $8.6(0.6)$ & $15.0(11.4)$ & 56 & $8.7(0.5)$ & $5.8(5.1)$ & 46 \\
\hline Ornoy, 2001 [20] Israel & $\begin{array}{l}\text { Conner's } \\
\text { (CG) }\end{array}$ & NR & $\begin{array}{l}\text { Benzodiazepines, } \\
\text { tobacco }\end{array}$ & No & $\mathrm{N} / \mathrm{A}$ & Heroin/MTD & $8.5(3.5)$ & $19.89(10.1)$ & 31 & $8.5(3.5)$ & $11.57(10.7)$ & 32 \\
\hline $\begin{array}{l}\text { Levine, } 2017 \text { [15] } \\
\text { New Zealand }\end{array}$ & $\begin{array}{l}\text { SDQ } \\
\text { (CG) }\end{array}$ & 88.2 & $\begin{array}{l}\text { Nicotine, cannabis, } \\
\text { benzodiazepine, other } \\
\text { opioid, stimulant, } \\
\text { antidepressant, alcohol }\end{array}$ & Yes & 20.6 & MTD & 4.5 (NR) & $4.29(2.4)$ & 87 & 4.5 (NR) & $2.44(2.1)$ & 103 \\
\hline $\begin{array}{l}\text { Wahlsten, } 2013 \text { [17] } \\
\text { Sweden }\end{array}$ & $\begin{array}{l}\text { SDQ } \\
\text { (TR) }\end{array}$ & 17.8 & $\begin{array}{l}\text { Relapse indicated, } \\
\text { substance not } \\
\text { mentioned }\end{array}$ & NA & 32 & BUP & $5.4(0.6)$ & $6.7(2.7)$ & 25 & $7.5(1.1)$ & $2.5(2.5)$ & 900 \\
\hline
\end{tabular}

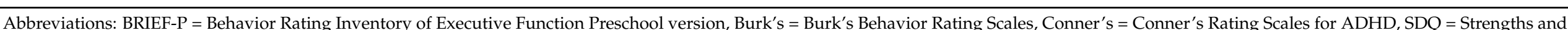

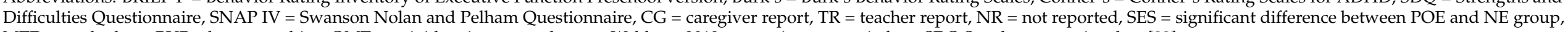
MTD = methadone, BUP = buprenorphine, OMT = opioid maintenance therapy. Wahlsten 2013 comparison group is from SDQ Sweden normative data [33]. 
Overall, three cohort studies were rated with 5.6 mean quality, ranging from 3 to 7 , and four case-control studies were rated 4.3 mean quality, ranging from 2 to 6 (see Table 3). Case control studies of low quality typically had less comparability of cases and controls due to study design or analysis, lower quality ascertainment of POE, different methods of ascertainment from cases and controls, or a different non-response rate [17,31]. The lower quality cohort study had poor comparability of cohorts based on the design or analysis and low-quality assessment of outcome [20].

Table 3. Quality assessment of included studies: Newcastle-Ottawa Scale.

\begin{tabular}{|c|c|c|c|c|}
\hline Cohort & Selection & Comparability & Outcome & Total \\
\hline $\begin{array}{l}\text { Nygaard, E., Slinning, K., Moe, V., } \\
\text { and Walhovd, K. B., } 2016 \text { [23] }\end{array}$ & 3 & 2 & 2 & 7 \\
\hline $\begin{array}{l}\text { Ornoy, A., Segal, J., Bar-Hamburger, } \\
\text { R., and Greenbaum, C., } 2001 \text { [20] }\end{array}$ & 2 & 0 & 1 & 3 \\
\hline $\begin{array}{l}\text { Levine, T. A., and Woodward, L. J., } \\
\qquad 2017 \text { [15] }\end{array}$ & 4 & 0 & 3 & 7 \\
\hline Case Control & Selection & Comparability & Exposure & Total \\
\hline $\begin{array}{l}\text { Konijnenberg, C., and Melinder, A., } \\
2015 \text { [32] }\end{array}$ & 2 & 2 & 1 & 5 \\
\hline $\begin{array}{l}\text { Davis, D. D., and Templer, D. } \\
\text { I.,1988 [21] }\end{array}$ & 3 & 1 & 2 & 6 \\
\hline Sandtorv et al., 2018 [31] & 2 & 2 & 0 & 4 \\
\hline Wahlsten and Sarman, 2013 [17] & 2 & 0 & 0 & 2 \\
\hline
\end{tabular}

\subsection{Behavioral Instruments}

The following instruments and subscales were used by the seven studies: the Strengths and Difficulties Questionnaire [hyperactivity/inattention subscale] [34], the Behavior Rating Inventory of Executive Function Preschool version [inhibit and working memory subscales] [35], Swanson Nolan and Pelham Questionnaire [hyperactivity/impulsivity items, and inattention items] [36], Achenbach Child Behavior Checklist [attention scale] [37], Burk's Behavior Rating Scales [attention and impulse control] [38], and Conner's Rating Scales for ADHD [combined symptoms] [39]. All behavioral scales included in this metaanalysis are rating scales commonly used in an ADHD diagnosis or measure symptoms of an ADHD diagnosis [40]. All instruments used in analyses were caregiver report, with the exception of one exclusive teacher report on the Strengths and Difficulties Questionnaire [17].

\subsection{Hyperactivity/Impulsivity Symptoms}

Three of the seven studies contained data specific to hyperactivity/impulsivity symptoms and were eligible for individual ADHD symptoms analysis. Thus, three studies were pooled to include 120 children with POE and 230 non-exposed children aging from 4.3 to 11.2 mean years. There was a significant positive association of POE with reported hyperactivity/impulsivity symptoms in childhood $(\mathrm{d}=1.40 ; 95 \% \mathrm{CI}, 0.49-2.31 ; p<0.003$; Figure 2). High heterogeneity was indicated $\left(\mathrm{I}^{2}=91.13\right)$; however, the number of studies was too small to perform a subgroup analysis. No studies rated as poor quality met inclusion for this analysis, indicated by scores above 3 on the Newcastle-Ottawa Scale. 


\begin{tabular}{lrrrrrrr} 
Study name & \multicolumn{7}{c}{ Statistics for each study } \\
& $\begin{array}{c}\text { Std diff } \\
\text { in means }\end{array}$ & $\begin{array}{c}\text { Lower } \\
\text { limit }\end{array}$ & $\begin{array}{c}\text { Standard } \\
\text { error }\end{array}$ & Variance & $\begin{array}{c}\text { Upper } \\
\text { limit }\end{array}$ & Z-Value & p-Value \\
Konijnenberg \& Melinder, 2015 & 0.909 & 0.401 & 0.259 & 0.067 & 1.417 & 3.509 & 0.000 \\
Davis \& Templer, 1988 & 1.019 & 0.462 & 0.284 & 0.081 & 1.576 & 3.587 & 0.000 \\
Sandtorv et al., 2018 & 2.221 & 1.858 & 0.185 & 0.034 & 2.583 & 12.007 & 0.000 \\
& 1.400 & 0.487 & 0.466 & 0.217 & 2.313 & 3.004 & 0.003
\end{tabular}

(a)

Studyname

$\begin{array}{lrc} & \begin{array}{c}\text { Std diff } \\ \text { in means }\end{array} & \begin{array}{c}\text { Standard } \\ \text { error }\end{array} \\ \text { Koninenberg \& Melinder, 2015 } & 0.776 & 0.256 \\ \text { Davis \& Templer, 1988 } & 1.493 & 0.302 \\ \text { Sandtor et al., 2018 } & 2.121 & 0.182 \\ \text { Nygaard et al., 2016 } & 0.979 & 0.208 \\ & 1.350 & 0.338\end{array}$

\section{Statistics for each study}

$\begin{array}{ccc}\text { Variance } & \begin{array}{c}\text { Lower } \\ \text { limit }\end{array} & \begin{array}{c}\text { Upper } \\ \text { limit }\end{array} \\ & 0224 & 127\end{array}$

Vimit z-Value p-Valu

$\begin{array}{lll}0.091 & 0.900 & 2.085\end{array}$

$\begin{array}{lll}0.033 & 1.764 & 2.479\end{array}$

$\begin{array}{lll}0.043 & 0.570 & 1.387\end{array}$

3.033
4.940
11.631
4.696
3.999

Std diff in means and $95 \% \mathrm{Cl}$

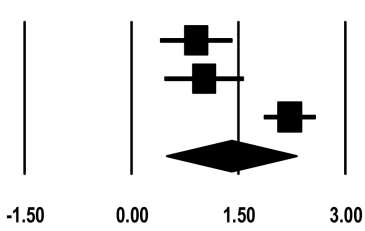

3.00
Std diff in means and $95 \% \mathrm{Cl}$

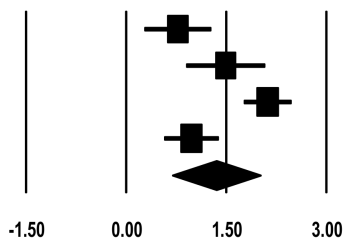

(b)

\begin{tabular}{lrrrrrrrr} 
Study name & \multicolumn{7}{c}{ Statistics for each study } \\
& $\begin{array}{c}\text { Std diff } \\
\text { in means }\end{array}$ & $\begin{array}{c}\text { Standard } \\
\text { error }\end{array}$ & $\begin{array}{c}\text { Variance } \\
\text { Lower }\end{array}$ & $\begin{array}{c}\text { limit } \\
\text { Koninnenberg \& Melinder, 2015 }\end{array}$ & $\begin{array}{c}\text { limit } \\
\text { Z-Value }\end{array}$ & p-Value \\
Davis \& Templer, 1988 & 0.840 & 0.257 & 0.066 & 0.336 & 1.344 & 3.266 & 0.001 \\
Sandtorv et al., 2018 & 1.282 & 0.293 & 0.086 & 0.706 & 1.857 & 4.368 & 0.000 \\
Nygaard et al., 2016 & 2.394 & 0.190 & 0.036 & 2.022 & 2.766 & 12.624 & 0.000 \\
Ornoy et al., 2001 & 1.009 & 0.211 & 0.045 & 0.595 & 1.423 & 4.777 & 0.000 \\
Levine \& Woodward, 2017 & 0.800 & 0.262 & 0.069 & 0.287 & 1.313 & 3.055 & 0.002 \\
Wahlsten \& Sarman, 2013 & 0.827 & 0.152 & 0.023 & 0.530 & 1.124 & 5.451 & 0.000 \\
& 1.676 & 0.206 & 0.043 & 1.272 & 2.081 & 8.119 & 0.000 \\
& 1.268 & 0.246 & 0.061 & 0.785 & 1.751 & 5.146 & 0.000
\end{tabular}

(c)

Figure 2. Forest plots comparing (a) hyperactivity/impulsivity, (b) inattention, and (c) combined ADHD subscales of children with POE versus non-exposed children. 


\subsection{Inattention Symptoms}

Of the seven studies, four had data specific to inattention symptoms and were eligible for individual ADHD symptom analysis. These four studies were pooled to include 177 children with POE and 277 non-exposed children ranging in age from 4.3 to 11.2 mean years. There was a significant positive association of POE with reported inattention symptoms in childhood ( $\mathrm{d}=1.35 ; 95 \% \mathrm{CI}, 0.69-2.01 ; p<0.0001)$ (Figure 2$)$. While high heterogeneity was present $\left(\mathrm{I}^{2}=88.30\right)$, the number of included studies was not sufficient to perform a subgroup analysis. No studies rated as poor quality met inclusion for this analysis, which was indicated by scores above 3 on the Newcastle-Ottawa Scale.

\subsection{Combined Subscale Symptoms}

A total of seven studies were pooled to include 319 children with POE and 1308 non-exposed children ranging in age from 4.3 to 11.2 mean years. There was a significant positive association of $\mathrm{POE}$ with reported combined ADHD subscale symptoms in childhood $(\mathrm{d}=1.27 ; 95 \% \mathrm{CI}, 0.79-1.75 ; p<0.0001)$ (Figure 2$)$. Heterogeneity analysis indicated high variation between studies $\left(\mathrm{I}^{2}=90.54\right)$. When removing studies of poor quality $[17,20]$, the significant positive association persisted $(\mathrm{d}=1.27,95 \% \mathrm{CI}, 0.63-1.91 ; p<0.0001)$.

Since ADHD symptoms vary by age, subgroup analyses were run for combined ADHD subscales for studies including a preschool age (4.3-4.5 mean years) and a school age (5.4-11.2 mean years). For the preschool subgroup, two studies were pooled to include 122 children with POE and 134 non-exposed children. There was a significant positive association of POE with reported combined ADHD subscale scores at the preschool age $(\mathrm{d}=0.83,95 \% \mathrm{CI}, 0.57-1.09 ; p<0.0001)$, with low heterogeneity $\left(\mathrm{I}^{2}=0\right)$, and no poor-quality studies (Figure 3).

For the school-age subgroup, five studies were pooled to include 197 children with POE and 1174 non-exposed children. There was a significant positive association of POE with reported subscale scores at the school age $(\mathrm{d}=1.45,95 \% \mathrm{CI}, 0.85-2.04 ; p<0.0001)$, with high heterogeneity $\left(\mathrm{I}^{2}=88.82\right)$ (Figure 3$)$. After removing low-quality studies $[17,20]$, the significant positive association persisted in school-aged children $(\mathrm{d}=1.57,95 \% \mathrm{CI}$, $0.64-2.50 ; p<0.0001)$, with high remaining heterogeneity $\left(\mathrm{I}^{2}=92.3\right)$.

Subgroup analyses were run for combined ADHD subscales for studies including healthy control/comparison groups (e.g., healthy children from mothers without OUD) and environmental control/comparison groups (e.g., children raised by a father with OUD or children raised in a low SES with environmental deprivation or neglect). For the healthy control/comparison subgroup, five studies were pooled to include 260 children with POE and 1251 non-exposed children. There was a significant positive association of POE with reported combined ADHD subscale scores amongst the healthy control/comparison subgroup $(\mathrm{d}=1.35,95 \% \mathrm{CI}, 0.73-1.97 ; p<0.0001)$, with high heterogeneity $\left(\mathrm{I}^{2}=92.19\right)$, and one poor-quality study (Figure 4). After removing the low-quality study [17], the significant positive association persisted amongst the healthy control/comparison subgroup $(\mathrm{d}=1.27$, $95 \% \mathrm{CI}, 0.50-2.04 ; p<0.0001)$, with high remaining heterogeneity $\left(\mathrm{I}^{2}=93.8\right)$. 
Study name

$\begin{array}{lrrrrrrr} & \begin{array}{c}\text { Std diff } \\ \text { in means }\end{array} & \begin{array}{c}\text { Standard } \\ \text { error }\end{array} & \text { Variance } & \begin{array}{c}\text { Lower } \\ \text { limit }\end{array} & \begin{array}{c}\text { Upper } \\ \text { limit }\end{array} & \text { Z-Value } & \text { p-Value } \\ \text { Konijnenberg \& Melinder, 2015 } & 0.840 & 0.257 & 0.066 & 0.336 & 1.344 & 3.266 & 0.001 \\ \text { Levine \& Woodward, 2017 } & 0.827 & 0.152 & 0.023 & 0.530 & 1.124 & 5.451 & 0.000 \\ & 0.830 & 0.131 & 0.017 & 0.574 & 1.086 & 6.355 & 0.000\end{array}$

Study name

Davis \& Templer, 1988

Sandtorv et al., 2018

Nygaard et al., 2016

Ornoy et al., 2001

Wahlsten \& Sarman, 2013

$\begin{array}{r}\text { Std diff } \\ \text { in means }\end{array}$
1.28
2.39
1.009
0.80
1.67
1.44

Statistics for each study

$\begin{gathered}\text { Standard } \\ \text { error }\end{gathered}$
0.293
0.190
0.211
0.262
0.206
0.304

$\begin{array}{rccrr}\text { Variance } & \begin{array}{c}\text { Limit } \\ \text { lime }\end{array} & \begin{array}{c}\text { Upper } \\ \text { limit }\end{array} & \text { Z-Value } & \text { p-Value } \\ 0.086 & 0.706 & 1.857 & 4.368 & 0.000 \\ 0.036 & 2.022 & 2.766 & 12.624 & 0.000 \\ 0.045 & 0.595 & 1.423 & 4.777 & 0.000 \\ 0.069 & 0.287 & 1.313 & 3.055 & 0.002 \\ 0.043 & 1.272 & 2.081 & 8.119 & 0.000 \\ 0.092 & 0.850 & 2.041 & 4.756 & 0.000\end{array}$

(a)

Std diff in means and $95 \% \mathrm{Cl}$

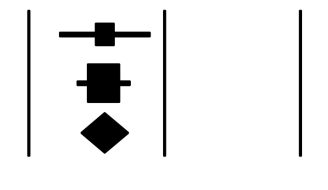

$-3.00$

$-1.50$

0.00

1.50

3.00

(b)
Std diff in means and $95 \% \mathrm{Cl}$

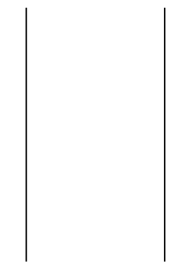

$-3.00$

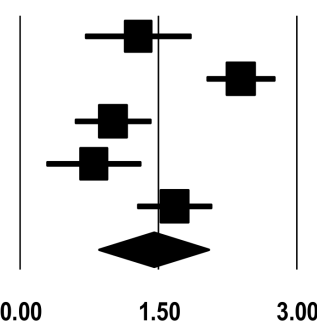

3.00

Figure 3. Forest plots comparing combined ADHD subscales of children with POE versus non-exposed children at the (a) preschool age and (b) school age. 


\section{Study name}

\begin{tabular}{|c|c|c|c|c|c|c|c|}
\hline & $\begin{array}{l}\text { Std diff } \\
\text { in means }\end{array}$ & $\begin{array}{l}\text { Standard } \\
\text { error }\end{array}$ & Variance & $\begin{array}{l}\text { Lower } \\
\text { limit }\end{array}$ & $\begin{array}{c}\text { Upper } \\
\text { limit }\end{array}$ & Z-Value & p-Value \\
\hline Wahlsten \& Sarman, 2013 & 1.676 & 0.208 & 0.043 & 1.272 & 2.081 & 8.119 & 0.000 \\
\hline Sandtorv et al., 2018 & 2.394 & 0.190 & 0.036 & 2.022 & 2.768 & 12.624 & 0.000 \\
\hline Levine \&. Woodward, 2017 & 0.827 & 0.152 & 0.023 & 0.530 & 1.124 & 5.451 & 0.000 \\
\hline onijnenberg \& Melinder, 2015 & 0.840 & 0.257 & 0.086 & 0.336 & 1.344 & 3.266 & 0.001 \\
\hline \multirow[t]{2}{*}{ Nygarard et al., 2016} & 1.009 & 0.211 & 0.045 & 0.595 & 1.423 & 4.777 & 0.000 \\
\hline & 1.353 & 0.317 & 0.101 & 0.731 & 1.974 & 4.284 & 0.000 \\
\hline
\end{tabular}

(a)

\section{Study name \\ Ornoy at a., 2001 \\ Davis \& Tempier, 1988}

\section{Statistics for each study}

\section{Std diff Standard Lower Upper} in means error Variance limit limit Z-Value p-Value

$\begin{array}{ll}1.292 & 0.293 \\ 0.200 & 0.262 \\ 1.023 & 0.240\end{array}$

$\begin{array}{lllll}0.006 & 0.706 & 1.857 & 4.368 & 0.000 \\ 0.009 & 0.287 & 1.313 & 3.055 & 0.002 \\ 0.058 & 0.552 & 1.493 & 4.260 & 0.000\end{array}$

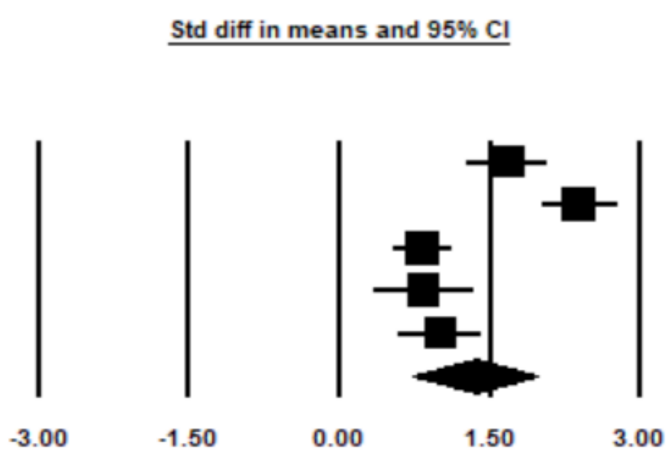

\section{diff in means and $95 \% \mathrm{C}$}

$-1.50$

\section{Std diff in means and $95 \% \mathrm{Cl}$}

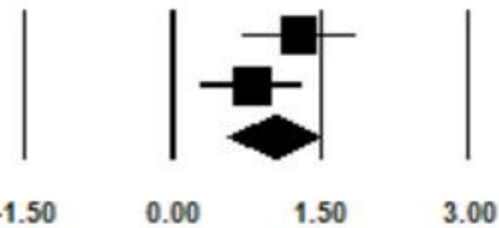

(b)

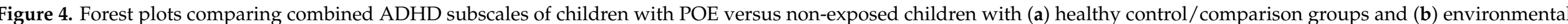
control/comparison groups. 
For the environmental control/comparison subgroup, two studies were pooled to include 59 children with POE and 60 non-exposed children. There was a significant positive association of $\mathrm{POE}$ with reported subscale scores amongst the environmental control/comparison subgroup $(\mathrm{d}=1.02,95 \% \mathrm{CI}, 0.55-1.49 ; p<0.0001)$, with low heterogeneity $\left(\mathrm{I}^{2}=33.28\right)$, and one poor-quality study [20] (Figure 4).

\subsection{Evaluation of Bias}

Due to an insufficient number of studies, publication bias was not assessed in the combined preschool $(n=2)$, combined environmental control/comparison $(n=2)$, and hyperactivity/impulsivity $(n=3)$ analyses. The Egger test and funnel plot review did not indicate publication bias for reported inattention (SE, 6.92; 95\% CI, $-42.45-28.61 ; p=0.49$ ), combined subscale of all children (SE, 5.95; 95\% CI, $-15.92-14.67 ; p=0.92)$, combined subscale of school-aged children (SE, $-11.12,95 \% \mathrm{CI}, 35.40-13.18 ; p=0.24$ ), or combined subscale of healthy control/comparison group (SE, 10.66; 95\% CI, $-31.84-36.01 ; p=0.86$ ) analyses.

\section{Discussion}

This study investigated the association of POE with ADHD symptoms in preschool and school-age children, both independently and combined. In alignment with our hypothesis, findings of this meta-analysis indicated that children with POE have higher hyperactivity/impulsivity, inattention, and combined ADHD symptoms compared to non-exposed controls. This positive association was apparent in children 4-14 years of age. Prenatal opioid exposure had the strongest association with hyperactivity/impulsivity, followed by inattention and then ADHD combined scores, although all effect sizes were considered large [27]. In addition, children with POE appeared to have more ADHD symptoms during school age compared to preschool age. The association of POE and ADHD symptoms seemed to be stronger when compared to healthy control/comparison groups, followed by environmental control/comparison groups.

These findings add to the existing literature suggesting that POE negatively impacts children's behavioral regulation in preschool and school-age children. While poor behavioral outcomes after POE have been reported [14], findings have been inconsistent or not included studies of older children [13]. Our analysis of data from seven published studies indicated that behavioral dysregulation is evident across childhood. Furthermore, this study adds to existing literature on the specific behavioral challenges of hyperactivity/impulsivity and inattention. While previous meta-analyses have examined behavioral outcomes after POE, none have tested ADHD symptoms specifically. Our study suggests that children with POE are more likely to experience hyperactivity/impulsivity, inattention, and combined symptoms. Moreover, while individual studies have documented elevated hyperactivity [22], impulsivity [21], or inattention [24] after POE, few studies have measured all symptoms of ADHD separately and combined. Thus, the current findings extend the emerging literature on behavioral dysregulation specific to ADHD symptoms in children with POE.

While results of our analyses suggest that ADHD symptoms in children with POE may increase as children age, there are a number of considerations. First, as the preschool subgroup analysis was constrained to two studies, interpretations reflect limited data. Second, all but one included study utilized caregiver report data. The literature suggests that caregivers of children with POE are more likely to have delayed identification of children's behavioral problems [23]. Thus, it is possible that the higher association with POE and ADHD symptoms at school age (versus preschool age) may be due to delayed identification of ADHD symptoms by caregivers. Third, the origin of this outcome is uncertain as cumulative biological, social, and environmental risk dynamically interact over time.

For instance, Barker's Fetal Origins of Adult Disease hypothesis states that human fetuses adapt to conditions in utero, which may program or permanently change fetal 
structure, affecting health over the life-course [41]. Accordingly, opioids may program the developing fetus, which then may interact with environmental and social vulnerabilities at later ages. The increased challenges in time may be related to a more complex and demanding social environment [23]. Longitudinal studies assessing ADHD symptoms indicated that children with POE persisted to have higher ADHD symptoms scores than non-exposed counterparts $[16,18,23]$. These studies also provided limited clarification on how factors such as maternal polydrug usage, type, dosing, and timing of opioid exposure, NAS diagnosis, change of caregiver, and SES may contribute to outcomes. Researchers speculate any challenges from the POE are further amplified by environmental, social, and biological risk factors [42]. Future studies should continue to investigate these relationships.

Although ADHD symptoms may present differently across developmental stages, their burden on individuals is typically chronic throughout the life-course [43]. Beyond the hallmark symptoms, preschool-aged children with ADHD are likely to have increased conflict with peers and lack of compliance with adults [44]. During school-age years, children with ADHD may experience a surge of oppositional behavior, academic problems, and conflicts with peers [44]. By adolescence, a rise in conflict with parents and emergence of high-risk behaviors are observed higher than the general population [44]. Furthermore, two-thirds of children with ADHD have a comorbid condition [45]. Thus, children with ADHD face greater adversity and stress compared to peers without ADHD [46]. If ADHD persists into adulthood, both personal and professional life may be disrupted [47]. Individuals with ADHD achieve lower educational levels, have higher unemployment rates, and are more likely to experience social relationship challenges than those without ADHD $[40,47,48]$.

Children with POE appeared to have more ADHD symptoms when compared to healthy controls instead of environmental controls, indicating that the postnatal rearing environment impacts the association between POE and ADHD symptoms. While the environmental controls face significant challenges similar to children with POE (e.g., low socio-economic status, father with OUD, environmental deprivation, neglect), the effect size among the environmental subgroup remained large [27], indicating that children with POE represent an exceptionally vulnerable population. This finding should be further explored by future researchers, as there were only two studies in the environmental subgroup analysis. Mothers of children with POE oftentimes have high rates of socio-economic difficulty and comorbid mental health challenges [12]. Many children with POE are at risk of child welfare concerns, with an average of one to two caregiver changes [11]. Children with POE have been removed from parental custody due to maternal substance abuse and mental health issues, child neglect, and maternal imprisonment and physical abuse [11], indicating several adverse childhood experiences. It is well documented that adverse childhood experiences have lasting impacts, including increased mental health problems, risky behaviors, infectious and chronic disease, while lowering education, occupation, and income opportunities [49-51].

Findings from this study indicate that children with POE are at high risk for ADHD symptoms that persist throughout childhood $[15,17,20,21,23,31,32]$. Results suggest that children with POE may benefit from long-term assistance, such as enhanced awareness and surveillance. Future researchers should continue to study long-term behavioral dysregulation after POE. When possible, studies should utilize gold-standard measurements to assess POE, such as urine analysis throughout pregnancy and meconium analysis after birth, as these methods for detecting in utero drug exposure are inexpensive, noninvasive, and will enhance understanding of exposure and outcome relationships [52]. Longitudinal studies should be carefully designed to properly assess biological, social, and environmental risk factors and report outcome data at each time point to increase the availability of aggregate analyses. Furthermore, researchers should report outcomes and subscale data of rating scales controlling for a host of demographic factors and health-related measures to adequately assess the phenotype of children with POE in relation to ADHD symptoms. Lastly, due to variability in behavioral rating scales, researchers investigating ADHD symp- 
toms should carefully review the literature to ensure rating scales match the psychometric properties of the DSM-IV ADHD diagnosis [53].

Strengths of this study include adhering to the recommended protocols related to meta-analyses, including independent reviewers, and achieving a good Cohen's $\mathrm{k}$ after title and abstract selection and full text retrieval stage. This meta-analysis explored symptoms of hyperactivity/impulsivity and inattention separately as well as combined, adding to the literature on the phenotype of children with POE and specific symptoms of ADHD. Regardless of variability of POE and rating scales, this study encompassed the breadth of available data and found consistent results. Moreover, our study investigated the longterm risk of POE and ADHD symptoms by including subgroup analyses of preschool and school-age children. Lastly, there were no indications of publication bias in any analyses, and sensitivity analyses were conducted, excluding low-quality studies.

While we contacted all primary authors for data, we had no responses with the requested data and were unable to include four pertinent studies for analyses $[21,22,24,54]$. Nonetheless, the studies excluded were conducted nearly thirty years ago [22], contained low sample sizes [21,22,54], or utilized a cross-sectional study design [22,54]. Thus, it is unlikely that effect sizes would have changed significantly as a result of the missing studies. All outcome data were self-reported from caregiver or teachers; however, while an ADHD diagnosis would be favorable to assess, behavioral scales are commonly used to assess ADHD in the literature [40,55]. Data extracted on biological and social risk were insufficient for further subgroup analyses, which may have explained the significant heterogeneity in analyses. Nonetheless, we attempted to account for this by using a random-effects meta-analysis. Two studies included were historical in nature [20,21]; however, the quality was ranked low only in one [20] of which sensitivity analyses were conducted, excluding low-quality studies. Lastly, the meta-analysis was restricted to English language upon review, which led to the exclusion of only one study that did not report the data needed for analysis.

\section{Conclusions}

This meta-analysis indicates that POE is significantly associated with ADHD symptoms across childhood. Results suggest an increased risk of ADHD symptoms during school age. These findings indicate children with POE may benefit from long-term assistance throughout childhood. Future research is needed to clarify the relationship between biological, environmental, social risk, and ADHD symptoms in children with POE.

Author Contributions: Conceptualization, A.N.S., L.M.R., K.W.K. and L.L.M.; methodology, A.N.S., L.M.R., L.L.M. and K.W.K.; software, A.N.S.; validation, A.N.S. and L.M.R.; formal analysis, A.N.S.; investigation, A.N.S. and L.M.R.; resources, A.N.S.; data curation, A.N.S. and L.M.R.; writingoriginal draft preparation, A.N.S.; writing—review and editing, A.N.S., L.M.R., K.W.K. and L.L.M.; visualization, A.N.S. and L.M.R.; supervision, L.L.M. and K.W.K.; project administration, K.W.K. and L.L.M.; All authors have read and agreed to the published version of the manuscript.

Funding: This research received no external funding.

Institutional Review Board Statement: Not applicable.

Informed Consent Statement: Not applicable.

Data Availability Statement: The data presented in this study are openly available in the published manuscripts cited within this study.

Acknowledgments: The authors have no acknowledgements.

Conflicts of Interest: The authors declare no conflict of interest. 


\section{Appendix A}

Table A1. PRISMA Checklist.

\begin{tabular}{|c|c|c|c|}
\hline Section/Topic & $\#$ & Checklist Item & Reported on Page \# \\
\hline \multicolumn{4}{|c|}{ TITLE } \\
\hline Title & 1 & $\begin{array}{l}\text { Identify the report as a systematic review, } \\
\text { meta-analysis, or both. }\end{array}$ & 1 \\
\hline \multicolumn{4}{|c|}{ ABSTRACT } \\
\hline $\begin{array}{l}\text { Structured } \\
\text { summary }\end{array}$ & 2 & $\begin{array}{l}\text { Provide a structured summary including, } \\
\text { as applicable: background; objectives; } \\
\text { data sources; study eligibility criteria, } \\
\text { participants, and interventions; study } \\
\text { appraisal and synthesis methods; results; } \\
\text { limitations; conclusions and implications } \\
\text { of key findings; systematic review } \\
\text { registration number. }\end{array}$ & 1 \\
\hline \multicolumn{4}{|c|}{ INTRODUCTION } \\
\hline Rationale & 3 & $\begin{array}{l}\text { Describe the rationale for the review in the } \\
\text { context of what is already known. }\end{array}$ & $1-2$ \\
\hline Objectives & 4 & $\begin{array}{l}\text { Provide an explicit statement of questions } \\
\text { being addressed with reference to } \\
\text { participants, interventions, comparisons, } \\
\text { outcomes, and study design (PICOS). }\end{array}$ & $2-3$ \\
\hline \multicolumn{4}{|c|}{ METHODS } \\
\hline $\begin{array}{l}\text { Protocol and } \\
\text { registration }\end{array}$ & 5 & $\begin{array}{c}\text { Indicate if a review protocol exists, if and } \\
\text { where it can be accessed (e.g., Web } \\
\text { address), and, if available, provide } \\
\text { registration information including } \\
\text { registration number. }\end{array}$ & 2 \\
\hline $\begin{array}{l}\text { Eligibility } \\
\text { criteria }\end{array}$ & 6 & $\begin{array}{l}\text { Specify study characteristics (e.g., PICOS, } \\
\text { length of follow-up) and report } \\
\text { characteristics (e.g., years considered, } \\
\text { language, publication status) used as } \\
\text { criteria for eligibility, giving rationale. }\end{array}$ & $2-3$ \\
\hline $\begin{array}{l}\text { Information } \\
\text { sources }\end{array}$ & 7 & $\begin{array}{c}\text { Describe all information sources (e.g., } \\
\text { databases with dates of coverage, contact } \\
\text { with study authors to identify additional } \\
\text { studies) in the search and date last } \\
\text { searched. }\end{array}$ & 3 \\
\hline Search & 8 & $\begin{array}{l}\text { Present full electronic search strategy for } \\
\text { at least one database, including any limits } \\
\text { used, such that it could be repeated. }\end{array}$ & 3 \\
\hline Study selection & 9 & $\begin{array}{l}\text { State the process for selecting studies (i.e., } \\
\text { screening, eligibility, included in } \\
\text { systematic review, and, if applicable, } \\
\text { included in the meta-analysis). }\end{array}$ & $2-5$ \\
\hline $\begin{array}{l}\text { Data collection } \\
\text { process }\end{array}$ & 10 & $\begin{array}{c}\text { Describe method of data extraction from } \\
\text { reports (e.g., piloted forms, independently, } \\
\text { in duplicate) and any processes for } \\
\text { obtaining and confirming data from } \\
\text { investigators. }\end{array}$ & $2-5$ \\
\hline
\end{tabular}


Table A1. Cont.

\begin{tabular}{|c|c|c|c|}
\hline Section/Topic & $\#$ & Checklist Item & Reported on Page \# \\
\hline Data items & 11 & $\begin{array}{l}\text { List and define all variables for which } \\
\text { data were sought (e.g., PICOS, funding } \\
\text { sources) and any assumptions and } \\
\text { simplifications made. }\end{array}$ & 3 \\
\hline $\begin{array}{l}\text { Risk of bias in } \\
\text { individual } \\
\text { studies }\end{array}$ & 12 & $\begin{array}{l}\text { Describe methods used for assessing risk } \\
\text { of bias of individual studies (including } \\
\text { specification of whether this was done at } \\
\text { the study or outcome level), and how this } \\
\text { information is to be used in any data } \\
\text { synthesis. }\end{array}$ & 3 \\
\hline $\begin{array}{l}\text { Summary } \\
\text { measures }\end{array}$ & 13 & $\begin{array}{l}\text { State the principal summary measures } \\
\text { (e.g., risk ratio, difference in means). }\end{array}$ & 3 \\
\hline $\begin{array}{l}\text { Synthesis of } \\
\text { results }\end{array}$ & 14 & $\begin{array}{l}\text { Describe the methods of handling data } \\
\text { and combining results of studies, if done, } \\
\text { including measures of consistency (e.g., } \mathrm{I}^{2} \text { ) } \\
\text { for each meta-analysis. }\end{array}$ & 3 \\
\hline $\begin{array}{l}\text { Risk of bias } \\
\text { across studies }\end{array}$ & 15 & $\begin{array}{c}\text { Specify any assessment of risk of bias that } \\
\text { may affect the cumulative evidence (e.g., } \\
\text { publication bias, selective reporting } \\
\text { within studies). }\end{array}$ & 3 \\
\hline $\begin{array}{l}\text { Additional } \\
\text { analyses }\end{array}$ & 16 & $\begin{array}{l}\text { Describe methods of additional analyses } \\
\text { (e.g., sensitivity or subgroup analyses, } \\
\text { meta-regression), if done, indicating } \\
\text { which were pre-specified. }\end{array}$ & 3 \\
\hline \multicolumn{4}{|c|}{ RESULTS } \\
\hline Study selection & 17 & $\begin{array}{l}\text { Give numbers of studies screened, } \\
\text { assessed for eligibility, and included in the } \\
\text { review, with reasons for exclusions at each } \\
\text { stage, ideally with a flow diagram. }\end{array}$ & $3-4$ \\
\hline $\begin{array}{l}\text { Study } \\
\text { characteristics }\end{array}$ & 18 & $\begin{array}{l}\text { For each study, present characteristics for } \\
\text { which data were extracted (e.g., study size, } \\
\text { PICOS, follow-up period) and provide the } \\
\text { citations. }\end{array}$ & $5-7$ \\
\hline $\begin{array}{l}\text { Risk of bias } \\
\text { within studies }\end{array}$ & 19 & $\begin{array}{l}\text { Present data on risk of bias of each study } \\
\text { and, if available, any outcome level } \\
\text { assessment (see item 12). }\end{array}$ & $8-11$ \\
\hline $\begin{array}{l}\text { Results of } \\
\text { individual } \\
\text { studies }\end{array}$ & 20 & $\begin{array}{l}\text { For all outcomes considered (benefits or } \\
\text { harms), present, for each study: (a) simple } \\
\text { summary data for each intervention group } \\
\text { (b) effect estimates and confidence } \\
\text { intervals, ideally with a forest plot. }\end{array}$ & $8-11$ \\
\hline $\begin{array}{l}\text { Synthesis of } \\
\text { results }\end{array}$ & 21 & $\begin{array}{c}\text { Present results of each meta-analysis done, } \\
\text { including confidence intervals and } \\
\text { measures of consistency. }\end{array}$ & $8-11$ \\
\hline $\begin{array}{l}\text { Risk of bias } \\
\text { across studies }\end{array}$ & 22 & $\begin{array}{l}\text { Present results of any assessment of risk of } \\
\text { bias across studies (see Item 15). }\end{array}$ & $8-11$ \\
\hline $\begin{array}{l}\text { Additional } \\
\text { analysis }\end{array}$ & 23 & $\begin{array}{l}\text { Give results of additional analyses, if done } \\
\text { (e.g., sensitivity or subgroup analyses, } \\
\text { meta-regression [see Item 16]). }\end{array}$ & $8-11$ \\
\hline
\end{tabular}


Table A1. Cont.

\begin{tabular}{|c|c|c|c|}
\hline Section/Topic & $\#$ & Checklist Item & Reported on Page \# \\
\hline \multicolumn{4}{|c|}{ DISCUSSION } \\
\hline $\begin{array}{l}\text { Summary of } \\
\text { evidence }\end{array}$ & 24 & $\begin{array}{l}\text { Summarize the main findings including } \\
\text { the strength of evidence for each main } \\
\text { outcome; consider their relevance to key } \\
\text { groups (e.g., healthcare providers, users, } \\
\text { and policy makers). }\end{array}$ & $11-13$ \\
\hline Limitations & 25 & $\begin{array}{l}\text { Discuss limitations at study and outcome } \\
\text { level (e.g., risk of bias), and at review level } \\
\text { (e.g., incomplete retrieval of identified } \\
\text { research, reporting bias). }\end{array}$ & 13 \\
\hline Conclusions & 26 & $\begin{array}{l}\text { Provide a general interpretation of the } \\
\text { results in the context of other evidence } \\
\text { and implications for future research. }\end{array}$ & $13-14$ \\
\hline \multicolumn{4}{|c|}{ FUNDING } \\
\hline Funding & 27 & $\begin{array}{l}\text { Describe sources of funding for the } \\
\text { systematic review and other support (e.g., } \\
\text { supply of data); role of funders for the } \\
\text { systematic review. }\end{array}$ & NA \\
\hline
\end{tabular}

\section{References}

1. Centers for Disease Control and Prevention. CDC Vital Signs: Prescription Painkiller Overdoses: A Growing Epidemic, Especially among Women; Centers for Disease Control and Prevention: Atlanta, GA, USA, 2017.

2. Ailes, E.C.; Dawson, A.L.; Lind, J.N.; Gilboa, S.M.; Frey, M.T.; Broussard, C.S.; Honein, M.A. Opioid prescription claims among women of reproductive age-United States, 2008-2012. Morb. Mortal. Wkly. Rep. 2015, 64, 37-41.

3. Patrick, S.W.; Davis, M.M.; Lehmann, C.U.; Cooper, W.O. Increasing incidence and geographic distribution of neonatal abstinence syndrome: United States 2009 to 2012. J. Perinatol. 2015, 35, 650-655. [CrossRef] [PubMed]

4. Cleary, B.J.; Donnelly, J.; Strawbridge, J.; Gallagher, P.J.; Fahey, T.; Clarke, M.; Murphy, D.J. Methadone dose and neonatal abstinence syndrome-systematic review and meta-analysis. Addiction 2010, 105, 2071-2084. [CrossRef] [PubMed]

5. Stover, M.W.; Davis, J.M. Opioids in pregnancy and neonatal abstinence syndrome. Semin. Perinatol. 2015, 39, 561-565. [CrossRef]

6. MacMullen, N.J.; Samson, L.F. Neonatal Abstinence Syndrome: An Uncontrollable Epidemic. Crit. Care Nurs. Clin. N. Am. 2018, 30, 585-596. [CrossRef]

7. $\quad$ Patrick, S.W.; Dudley, J.; Martin, P.R.; Harrell, F.E.; Warren, M.D.; Hartmann, K.E.; Ely, E.W.; Grijalva, C.G.; Cooper, W.O. Prescription opioid epidemic and infant outcomes. Pediatrics 2015, 135, 842-850. [CrossRef] [PubMed]

8. Allocco, E.; Melker, M.; Rojas-Miguez, F.; Bradley, C.; Hahn, K.A.; Wachman, E.M. Comparison of Neonatal Abstinence Syndrome Manifestations in Preterm Versus Term Opioid-Exposed Infants. Adv. Neonatal Care 2016, 16, 329-336. [CrossRef] [PubMed]

9. Monnelly, V.J.; Anblagan, D.; Quigley, A.; Cabez, M.B.; Cooper, E.S.; Mactier, H.; Semple, S.I.; Bastin, M.E.; Boardman, J.P. Prenatal methadone exposure is associated with altered neonatal brain development. NeuroImage Clin. 2018, 18, 9-14. [CrossRef]

10. Roussos-Ross, K.; Reisfield, G.; Elliot, I.; Dalton, S.; Gold, M. Opioid use in pregnant women and the increase in neonatal abstinence syndrome: What is the cost? J. Addict. Med. 2015, 9, 222-225. [CrossRef]

11. Lean, R.E.; Pritchard, V.E.; Woodward, L.J. Child protection and out-of-home placement experiences of preschool children born to mothers enrolled in methadone maintenance treatment during pregnancy. Child. Youth Serv. Rev. 2013, 35, 1878-1885. [CrossRef]

12. Davie-Gray, A.; Moor, S.; Spencer, C.; Woodward, L.J. Psychosocial characteristics and poly-drug use of pregnant women enrolled in methadone maintenance treatment. Neurotoxicol. Teratol. 2013, 38, 46-52. [CrossRef] [PubMed]

13. Baldacchino, A.; Arbuckle, K.; Petrie, D.J.; McCowan, C. Erratum: Neurobehavioral consequences of chronic intrauterine opioid exposure in infants and preschool children: A systematic review and meta-analysis. BMC Psychiatry 2015, 15, 134. [CrossRef]

14. Monnelly, V.J.; Hamilton, R.; Chappell, F.M.; Mactier, H.; Boardman, J.P. Childhood neurodevelopment after prescription of maintenance methadone for opioid dependency in pregnancy: A systematic review and meta-analysis. Dev. Med. Child Neurol. 2019, 61, 750-760. [CrossRef]

15. Levine, T.A.; Woodward, L.J. Early inhibitory control and working memory abilities of children prenatally exposed to methadone. Early Hum. Dev. 2017, 116, 68-75. [CrossRef]

16. Slinning, K. Foster placed children prenatally exposed to poly-substances-attention-related problems at ages 2 and $41 / 2$. Eur. Child Adolesc. Psychiatry 2004, 13, 19-27. [CrossRef] [PubMed]

17. Sundelin Wahlsten, V.; Sarman, I. Neurobehavioural development of preschool-age children born to addicted mothers given opiate maintenance treatment with buprenorphine during pregnancy. Acta Paediatr. 2013, 102, 544-549. [CrossRef] 
18. Ornoy, A. The impact of intrauterine exposure versus postnatal environment in neurodevelopmental toxicity: Long-term neurobehavioral studies in children at risk for developmental disorders. Toxicol. Lett. 2003, 140-141, 171-181. [CrossRef]

19. Ornoy, A.; Daka, L.; Goldzweig, G.; Gil, Y.; Mjen, L.; Levit, S.; Shufman, E.; Bar-Hamburger, R.; Greenbaum, C.W. Neurodevelopmental and psychological assessment of adolescents born to drug-addicted parents: Effects of SES and adoption. Child Abus. Negl. 2010, 34, 354-368. [CrossRef] [PubMed]

20. Ornoy, A.; Segal, J.; Bar-Hamburger, R.; Greenbaum, C. Developmental outcome of school-age children born to mothers with heroin dependency: Importance of environmental factors. Dev. Med. Child Neurol. 2001, 43, 668-675. [CrossRef] [PubMed]

21. Davis, D.D.; Templer, D.I. Neurobehavioral functioning in children exposed to narcotics in utero. Addict. Behav. 1988, 13, 275-283. [CrossRef]

22. Cubas, M.M.; Field, T. Children of methadone-dependent women: Developmental outcomes. Am. J. Orthopsychiatry 1993, 63, 266-276. [CrossRef]

23. Nygaard, E.; Slinning, K.; Moe, V.; Walhovd, K.B. Behavior and Attention Problems in Eight-Year-Old Children with Prenatal Opiate and Poly-Substance Exposure: A Longitudinal Study. PLoS ONE 2016, 11, e0158054. [CrossRef]

24. Hickey, J.E.; Suess, P.E.; Newlin, D.B.; Spurgeon, L.; Porges, S.W. Vagal tone regulation during sustained attention in boys exposed to opiates in utero. Addict. Behav. 1995, 20, 43-59. [CrossRef]

25. Yeoh, S.L.; Eastwood, J.; Wright, I.M.; Morton, R.; Melhuish, E.; Ward, M.; Oei, J.L. Cognitive and Motor Outcomes of Children with Prenatal Opioid Exposure: A Systematic Review and Meta-analysis. JAMA Netw. Open 2019, 2, e197025. [CrossRef]

26. Shamseer, L.; Moher, D.; Clarke, M.; Ghersi, D.; Liberati, A.; Petticrew, M.; Shekelle, P.; Stewart, L.A. Preferred reporting items for systematic review and meta-analysis protocols (PRISMA-P) 2015: Elaboration and explanation. BMJ 2015, 349, g7647. [CrossRef] [PubMed]

27. Borenstein, M.; Hedges, L.V.; Higgins, J.P.T.; Rothstein, H.R. Introduction to Meta-Analysis; John Wiley \& Sons Ltd.: West Sussex, UK, 2009.

28. Wells, G.A.; Shea, B.; O'Connell, D.; Robertson, J.; Peterson, J.; Welch, V.; Losos, M.; Tugwell, P. The Newcastle-Ottawa Scale (NOS) for Assessing the Quality of Nonrandomized Studies in MetaAnalysis; Ottawa Health Research Institute: Ottawa, ON, Canada, 1999.

29. Walhovd, K.B.; Moe, V.; Slinning, K.; Due-Tonnessen, P.; Bjornerud, A.; Dale, A.M.; van der Kouwe, A.; Quinn, B.T.; Kosofsky, B.; Greve, D.; et al. Volumetric cerebral characteristics of children exposed to opiates and other substances in utero. NeuroImage 2007, 36, 1331-1344. [CrossRef] [PubMed]

30. Ornoy, A.; Michailevskaya, V.; Lukashov, I.; Bar-Hamburger, R.; Harel, S. The developmental outcome of children born to heroin-dependent mothers, raised at home or adopted. Child Abus. Negl. 1996, 20, 385-396. [CrossRef]

31. Sandtorv, L.B.; Fevang, S.K.E.; Nilsen, S.A.; Boe, T.; Gjestad, R.; Haugland, S.; Elgen, I.B. Symptoms Associated with Attention Deficit/Hyperactivity Disorder and Autism Spectrum Disorders in School-Aged Children Prenatally Exposed to Substances. Subst. Abus. 2018, 12, 1178221818765773. [CrossRef]

32. Konijnenberg, C.; Melinder, A. Executive function in preschool children prenatally exposed to methadone or buprenorphine. Child Neuropsychol. 2015, 21, 570-585. [CrossRef] [PubMed]

33. Smedje, H.; Broman, J.E.; Hetta, J.; von Knorring, A.L. Psychometric properties of a Swedish version of the Strengths and Difficulties Questionnaire. Eur. Child Adolesc. Psychiatry 1999, 8, 63-70. [CrossRef] [PubMed]

34. Goodman, R.; Ford, T.; Richards, H.; Gatward, R.; Meltzer, H. The Development and Well-Being Assessment: Description and initial validation of an integrated assessment of child and adolescent psychopathology. J. Child Psychol. Psychiatry 2000, 41, 645-655. [CrossRef]

35. Gioia, G.A.; Espy, K.A.; Isquith, P.K. BRIEF-P Behavior Rating Inventory of Executive Function-Preschool Version: Professional Manual; Psychological Assessment Resources Inc.: Lutz, FL, USA, 2003.

36. Bussing, R.; Fernandez, M.; Harwood, M.; Hou, W.; Garvan, C.W.; Eyberg, S.M.; Swanson, J.M. Parent and Teacher SNAP-IV Ratings of Attention Deficit Hyperactivity Disorder Symptoms: Psychometric Properties and Normative Ratings From a School District Sample. Assessment 2008, 15, 317-328. [CrossRef] [PubMed]

37. Achenbach, T.M. The Child Behavior Profile: I. Boys aged 6-11. J. Consult. Clin. Psychol. 1978, 46, 478-488. [CrossRef]

38. Burks, H. Burks Behavior Rating Scales; Western Psychological Services: Los Angeles, CA, USA, 1975.

39. Conners, C.K.; Sitarenios, G.; Parker, J.D.A.; Epstein, J.N. The Revised Conners' Parent Rating Scale (CPRS-R): Factor Structure, Reliability, and Criterion Validity. J. Abnorm. Child Psychol. 1998, 26, 257-268. [CrossRef] [PubMed]

40. Mueller, K.L.; Tomblin, J.B. Diagnosis of ADHD and its Behavioral, Neurologic and Genetic Roots. Top. Lang. Disord. 2012, 32, 207-227. [CrossRef]

41. Barker, D.J. In utero programming of chronic disease. Clin. Sci. 1998, 95, 115-128. [CrossRef]

42. Maguire, D.J.; Taylor, S.; Armstrong, K.; Shaffer-Hudkins, E.; Germain, A.M.; Brooks, S.S.; Cline, G.J.; Clark, L. Long-Term Outcomes of Infants with Neonatal Abstinence Syndrome. Neonatal Netw. 2016, 35, 277-286. [CrossRef] [PubMed]

43. Mahone, E.M.; Denckla, M.B. Attention-Deficit/Hyperactivity Disorder: A Historical Neuropsychological Perspective. J. Int. Neuropsychol. Soc. 2017, 23, 916-929. [CrossRef] [PubMed]

44. Koumoula, A. The course of attention deficit hyperactivity disorder (ADHD) over the life span. Psychiatr. Psychiatr. 2012, 23, 49-59.

45. Larson, K.; Russ, S.A.; Kahn, R.S.; Halfon, N. Patterns of comorbidity, functioning, and service use for US children with ADHD, 2007. Pediatrics 2011, 127, 462-470. [CrossRef] 
46. Humphreys, K.L.; Zeanah, C.H. Deviations from the Expectable Environment in Early Childhood and Emerging Psychopathology. Neuropsychopharmacology 2015, 40, 154-170. [CrossRef]

47. Harpin, V.A. The effect of ADHD on the life of an individual, their family, and community from preschool to adult life. Arch. Dis. Child. 2005, 90, i2-i7. [CrossRef] [PubMed]

48. Kessler, R.C.; Adler, L.; Ames, M.; Barkley, R.A.; Birnbaum, H.; Greenberg, P.; Johnston, J.A.; Spencer, T.; Ustun, T.B. The prevalence and effects of adult attention deficit/hyperactivity disorder on work performance in a nationally representative sample of workers. J. Occup. Environ. Med. 2005, 47, 565-572. [CrossRef] [PubMed]

49. Bethell, C.D.; Simpson, L.A.; Solloway, M.R. Child Well-being and Adverse Childhood Experiences in the United States. Acad. Pediatr. 2017, 17, S1-S3. [CrossRef] [PubMed]

50. Kalmakis, K.A.; Chandler, G.E. Health consequences of adverse childhood experiences: A systematic review. J. Am. Assoc. Nurse Pract. 2015, 27, 457-465. [CrossRef] [PubMed]

51. Hughes, K.; Bellis, M.A.; Hardcastle, K.A.; Sethi, D.; Butchart, A.; Mikton, C.; Jones, L.; Dunne, M.P. The effect of multiple adverse childhood experiences on health: A systematic review and meta-analysis. Lancet Public Health 2017, 2, e356-e366. [CrossRef]

52. Kocherlakota, P. Neonatal abstinence syndrome. Pediatrics 2014, 134, e547-e561. [CrossRef]

53. Collett, B.R.; Ohan, J.L.; Myers, K.M. Ten-year review of rating scales. V: Scales assessing attention-deficit/hyperactivity disorder. J. Am. Acad. Child. Adolesc. Psychiatry 2003, 42, 1015-1037. [CrossRef]

54. Finger, B.; Jobin, A.; Bernstein, V.J.; Hans, S. Parenting contributors to early emerging problem behaviour in children of mothers in methadone maintenance treatment. Infant Child Dev. 2018, 27, e2042. [CrossRef]

55. Chang, L.Y.; Wang, M.Y.; Tsai, P.S. Diagnostic Accuracy of Rating Scales for Attention-Deficit/Hyperactivity Disorder: A Metaanalysis. Pediatrics 2016, 137, e20152749. [CrossRef] [PubMed] 